\title{
Automatic Robust Medical Image Registration Using a New Democratic Vector Optimization Approach with Multiple Measures
}

\author{
Matthias Wacker ${ }^{1,2}$ and Frank Deinzer ${ }^{2,3}$ \\ 1 Institute of Medical Statistics, Computer Sciences and Documentation, \\ Friedrich-Schiller-University of Jena, GER \\ matthias.wacker@mti.uni-jena.de \\ 2 Siemens AG, Healthcare Sector, Forchheim, GER \\ 3 University of Applied Sciences Würzburg-Schweinfurt, GER \\ frank.deinzer@fhws.de
}

\begin{abstract}
The registration of various data is a challenging task in medical image processing and a highly frequented area of research. Most of the published approaches tend to fail sporadically on different data sets. This happens due to two major problems. First, local optimization strategies induce a high risk when optimizing nonconvex functions. Second, similarity measures might fail if they are not suitable for the data. Thus, researchers began to combine multiple measures by weighted sums. In this paper, we show severe limitations of such summation approaches. We address both issues by a gradient-based vector optimization algorithm that uses multiple similarity measures. It gathers context information from the iteration process to detect and suppress failing measures. The new approach is evaluated by experiments from the field of 2D-3D registration. Besides its generic character with respect to arbitrary data, the main benefit is a highly robust iteration behavior, where even very poor initial guesses of the transform result in good solutions.
\end{abstract}

\section{Introduction}

Image registration can be described as the task of finding a transformation from the coordinate system of one image into another in a way that corresponding information is aligned [1]. This challenging task is a highly frequented area of research and subject of numerous publications in the last 15 years [2 3415]. Dependend on the application ranging from neuro surgery to radio therapy and many more, the requirements include a high accuracy, time efficiency and robustness which are of course highly conflictive.

State of the art approaches use similarity measures that try to model the quality of the registration, given a certain transformation. Based on this objective function an optimization scheme is applied to search for the best transform. So there exist two main reasons for a failed registration. First, the optimization algorithm may get stuck in a wrong local optimum. This problem can be handled up to a certain extent by more complex search strategies at the cost 
of computational time [6]. Second, similarity measures can simply fail, what is often overseen. It means that the measure has its extremum at a location in the parameter space that does not correspond to meaningful registration. Although Penney et al. found already in $1998[3$ that different measures can lead to different results, this problem has hardly been further considered.

In order to avoid the problem of failing measures, researchers constructed more complex ones [7] and started to build convex combinations of two or more similarity formulations [4]. Feldmann et al. 8] used the idea of democratic integration of Triesch et al. 9], where an arbitrary number of measures are used simultaneously in a dynamically weighted sum. Since summation approaches have severe limitations as they suffer from scaling issues, it can be said that the overall approach to tackle that aspect has not been found yet.

In this paper we design a new iterative vector optimization scheme that will allow for an efficient and robust registration. It works with a set of similarity measures that are all treated equally so that the highly different scaling of the single measures does not affect their influence compared to the remaining ones. For detecting failing measures, the necessary context information can be extracted from the iteration trajectory and is used to suppress their influence.

First of all, section 2 will give a motivation of the algorithm design, followed by the formal definition. Secondly, section 3 demonstrates the capabilities of the method in two experiments. The paper closes with a discussion in section 4

\section{Method}

In general, similarity measures are highly nonconvex functions with unpredictable response behavior to parameter changes. Therefore, they are hard to normalize. Although it is mostly possible to give theoretical lower and upper bounds, their actually used range is not known in advance. But we know by design that all similarity measures try to characterize the point of optimal registration what implies that they should share an extremum.

The working hypotheses of the presented approach assumes the following situation. A subset of the similarity measures works quite well, once the parameters are close enough to the solution. The rest of the measures fails to give useful information. These subsets depend on the data set and dynamically on the current guess of the parameters which may be out- or inside the region of convergence for some measures.

\subsection{Motivation}

For a start, we give a brief demonstration about the problem of summation approaches. Figure 1 shows three different functions on the left side and their sum on the right side. Two of the three functions share an optimal point - the sought solution. Beneath the functions, arrows denote the iteration directions $d\left(f_{i}\right)$ a gradient descent method would suggest. On the right side, the failing function obviously dominates in the sum $d(f)_{\Sigma}$. But instead of building the 

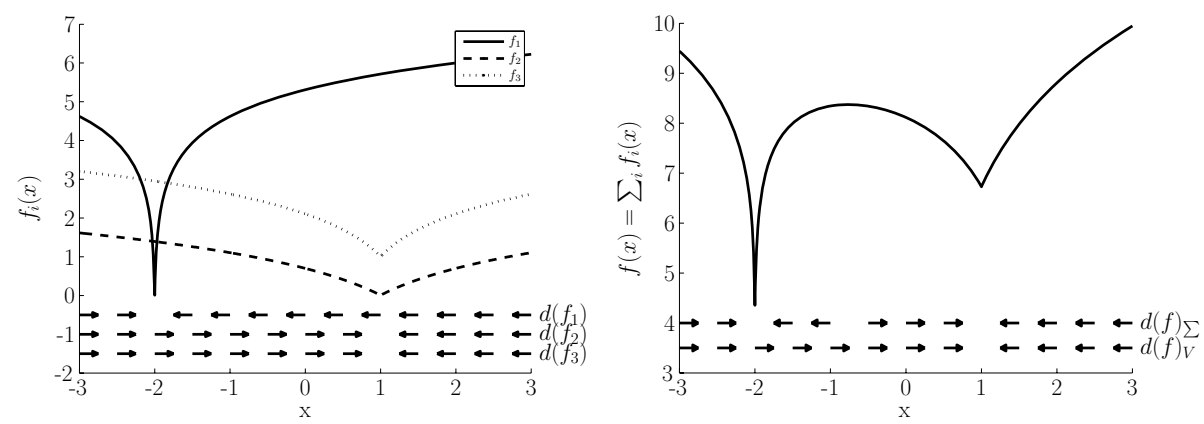

Fig. 1. Iteration directions of a gradient descent approach on single measures and a sum of measures compared to voting

gradient of the sum we could take the similarities as vector valued information. The gradient of each component of this vector votes for an iteration direction to build a majority decision. The result $d(f)_{V}$ is shown in the second row of arrows on the right side. Note that the algorithm would iterate right through the wrong minimum to the correct solution. Be also aware of the fact, that a multiplication of the wrong function with an arbitrary large number would still give the same voting result while the summation approach would be lost.

Considering n-dimensional problems, we recognize that there is an infinite set of directions to vote for. Of course, this is an ill posed task. Hence, we have to reinterpret the voting of a direction candidate as an averaging of the direction suggestions. The bad side effect of this averaging is that failing measures have a disturbing effect. But assuming we know which measures currently fail, we can easily suppress their influence by a small weighting factor. In terms of optimization, this failing can easily be described: let the measure be a function that has to be minimized, and let us assume we have already a previous iteration. If the last iteration was locally an ascend direction for the measure, it would have preferred the opposing direction and by that voted against the chosen one. This simple observation will be the core idea of the following method.

\subsection{Registration by Vector Optimization}

In the field of vector optimization, Edgeworth-Pareto (EP) optimality is defined with respect to an ordering cone [10. In general, the set of EP-optimal points is of infinite cardinality. To define a single solution, we incorporate the working hypothesis by a diagonal matrix $I$ with rank deficiency. It selects the working measures which share an optimal point $\hat{\boldsymbol{x}}$ and by that, characterize a strong EPoptimal point for this reduced sub problem. Associating with $\hat{\boldsymbol{x}}$ the maximum rank of $I$ (namely $\operatorname{rank}(\hat{\boldsymbol{x}})$ ), for which $\hat{\boldsymbol{x}}$ is still a strong EP-optimal point, we write the registration problem as

$$
\max _{I \in \mathbb{R}^{m \times m}}\left\{\operatorname{rank}\left(\underset{\boldsymbol{x} \in \mathbb{R}^{n}}{\arg \min } f_{I}(\boldsymbol{x})\right)\right\} \text {, where } f_{I}(\boldsymbol{x}):=I \cdot f(\boldsymbol{x}), \quad f(\boldsymbol{x}) \in \mathbb{R}^{m} .
$$




\subsection{Algorithm Design}

Our iterative optimization algorithm that realizes (1) will repeat the same basic steps as classical approaches: determine an iteration direction $\boldsymbol{d}$ and a step width $\lambda$. The presented method will use a scale parameter $\delta$ that gets reduced when oscillating behavior occurs. As it is straightforward, we omited the embedding in a multiresolution approach.

Iteration Direction. To compute the iteration direction, we approximate gradient information by central differences with evaluations of the functions in a distance of $\delta$. To create a democratic approach where each measure $f_{i}$ has an equal influence independent from its scaling, we reduce the gradient to directional information as a vector with unit length. Without loss of generality we assume that all measures have to be minimized. The continuous equivalent for the weighted discrete vote idea can be formalized as weighted averaging of the negated normalized gradients

$$
\boldsymbol{d}(\boldsymbol{x})=-\frac{1}{N} \sum_{i} w_{i} \nabla_{\|\cdot\|} f_{i}(\boldsymbol{x}), \text { where } \nabla_{\|\cdot\|} f_{i}(\boldsymbol{x}):=\frac{\nabla f_{i}(\boldsymbol{x})}{\left\|\nabla f_{i}(\boldsymbol{x})\right\|} .
$$

For the moment, the weights $w_{i}$ should be considered as given, because they will be described after presenting the framework.

Step Size. In order to determine a step size, usually a one dimensional optimization is applied. A weighted sum of all measures would be a possible objective function for that. But the above example already revealed the disadvantages of scalar valued approaches. In account of that, we define a rough but robust method: the scale parameter $\delta$ of the algorithm suggests two candidates for the step size $\lambda_{1}=\delta$ and $\lambda_{2}=2 \cdot \delta$. Between the two candidates, a discrete weighted vote is performed, that is summing up the weights of the measures that would prefer $\lambda_{1}$ and comparing it with the corresponding value of $\lambda_{2}$ where the larger value marks the result.

Adaption of the Weight Factors. Changing the weights in (2) during optimization results in a dynamically varying shape of the ordering cone realized by the linear mapping $I$ in (1). Adapting the weights is the most crucial task in the algorithm. So we have to design an automatism that makes the weighting strategy meet our requirements. In this approach, the weights are generated by multiplication of two terms

$$
w_{i}=w_{i, R E} \cdot w_{i, C O},
$$

that are described in the following.

Reliability of a Measure $\left(w_{i, R E}\right)$. To detect failing measures, we observe over time how often a measure gives a useful contribution to the iteration. Therefore, we define a history of length $h$ to realize a time limited context extraction from the current iteration $t_{c}$ to $t_{c-h}$. Within this context we determine to which extent the iteration direction was a descend direction for the measure. This can be formalized as having an angular of less than $\frac{\pi}{2}$ from the negated gradient 


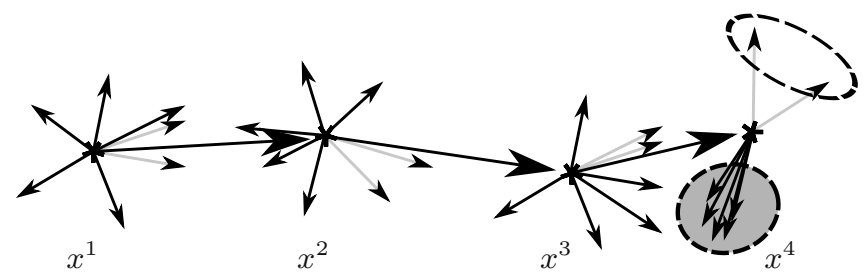

Fig. 2. Problem of inertia of reliability weights

direction to the iteration direction. A normalization is applied to keep the weights in $[0,1]$. The angular between vectors $a$ and $b$ is denoted by $\Varangle(a, b)$

$$
w_{i, R E}^{1}=\frac{\#\left\{t \mid \Varangle\left(-\nabla_{\|\cdot\|} f_{i}^{t}, d^{t}\right)<\frac{\pi}{2}, t \in\left\{t_{c-1}, t_{c-2}, \ldots, t_{c-h}\right\}\right\}}{\sum_{j} \#\left\{t \mid \Varangle\left(-\nabla_{\|\cdot\|} f_{j}^{t}, d^{t}\right)<\frac{\pi}{2}, t \in\left\{t_{c-1}, t_{c-2}, \ldots, t_{c-h}\right\}\right\}} .
$$

As a random similarity measure could get relative high weight, we penalize volatile gradient changes with a multiplication ramp. The most 'random' measure gets multiplied by one and the least random measure gets multiplied with $\gamma>1$ to get a $[1, \gamma]$ normalization.

$$
\begin{gathered}
\tilde{w}_{i, R E}^{2}=\sum_{t_{k} \in\left\{t_{c}, \ldots, t_{c-h}\right\}} \Varangle\left(\nabla_{\|\cdot\|} f_{i}^{t_{k}}, \nabla_{\|\cdot\|} f_{i}^{t_{k-1}}\right) \\
w_{i, R E}^{2}=\gamma-(\gamma-1) \cdot \frac{\tilde{w}_{i, R E}^{2}-\min _{j} \tilde{w}_{j, R E}^{2}}{\max _{l} \tilde{w}_{l, R E}^{2}-\min _{j} \tilde{w}_{j, R E}^{2}}
\end{gathered}
$$

Together with $w_{i, R E}^{1}$ we get the total reliability in the given time context

$$
w_{i, R E}=w_{i, R E}^{1} \cdot w_{i, R E}^{2}
$$

Conformity of a Measure $\left(w_{i, C O}\right)$. The reliability weight can introduce a problem of inertia as the cardinal numbers in (44) can change only by one in each iteration. Figure 2 shows four following iterations $x^{1}, \ldots, x^{4}$ with the negated gradients of the measures. The preferred directions of the measures differ a lot in the first three steps. Two measures (gray) point every time in iteration direction and get therefore a high reliability weight. In the fourth iteration all except these two measures point into the same direction but the reliability could make the algorithm iterate in the opposing direction. Thus, we also consider the unweighted mean of negated gradients. Measures that point closely in that direction are conform with the majority in the current situation.

$$
w_{i, C O}=\frac{\pi-\Varangle\left(\nabla_{\|\cdot\|} f_{i}^{t_{c}}, \sum_{\nu} \nabla_{\|\cdot\|} f_{\nu}^{t_{c}}\right)}{\sum_{j} \pi-\Varangle\left(\nabla_{\|\cdot\|} f_{j}^{t_{c}}, \sum_{\nu} \nabla_{\|\cdot\|} f_{\nu}^{t_{c}}\right)} .
$$

Subtraction from the maximum angle $\pi$ and division gives again a $[0,1]$ normalization of the weights.

\section{Experiments and Results}

The used data was acquired from three different phantoms (skull, pelvis and thigh) with a Siemens AXIOM Artis dFA C-arm device. To get a rigid 2D-3D 


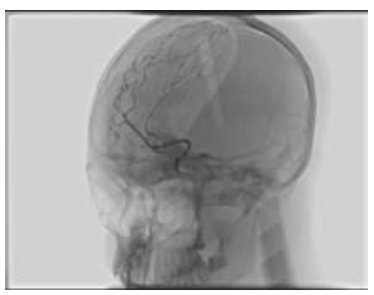

(a)

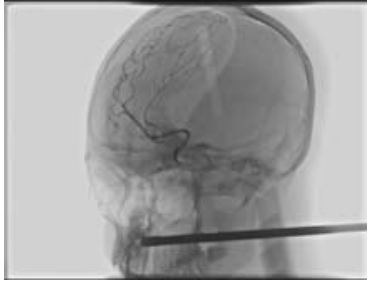

(b)

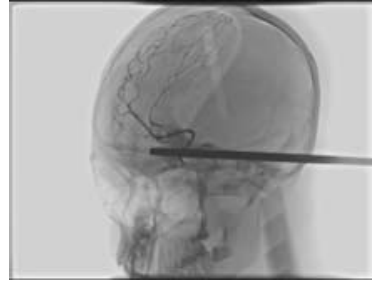

(c)

Fig. 3. Image data without (a) and with (b),(c) disturbing instruments present

registration problem, we reconstructed $3 \mathrm{D}$ volumes of $512 \times 512 \times 392$ voxels and reduced them to half size, took images of size $1024 \times 1024$ and resampled them to a resolution of $256 \times 256$. In the following, all error values represent the mean projected distance of corresponding points (equally spaced grid points in the center $\left(\frac{1}{3}\right)^{3}$-th volume) in $\mathrm{mm}$ on the detector plane. As the phantoms were not moved between reconstruction and image acquisition the system protocol could be used as ground truth information. Due to the limited precision of the C-arm device, the error values have a disturbance in the range of a few mm. As setup for the presented algorithm we chose $h=5, \gamma=2$ (6) and the following set of similarity measures: energy of differences (ED), gradient correlation $(G C)$, gradient differences (GD), joint entropy (JE), mutual information (MI), normalized cross correlation (NCC), pattern intensity (PI), ratio of image uniformity (RIU) and sum of squared differences (SSD) (see [3.5] and references).

\subsection{Robustness to Heavily Disturbing Image Contents}

In the first experiment, we simulate surgical instruments by adding a screwdriver to the skull phantom after it has been reconstructed. Some measures are sensitive to such disturbing image content. Figure 3 shows the original phantom without and with the instrument for two different positions. We compare the presented approach with best-neighbor $(\mathrm{BN})$ optimization approaches of single similarity measures. We generated 10 random initial parameter sets (3D rigid transforms) in the range of $19 \mathrm{~mm}$ to $42 \mathrm{~mm}$ distance to the optimal solution leading to the results, shown in Table 1 On this data set, MI and PI failed, gradient correlation worked quite well in the case of (a) but its error increases by almost $1 \mathrm{~cm}$ in the problem (b), The presented voting approach shows only a minimum increase of $0.1 \mathrm{~mm}$. Although the the error values appear high, what is due to the mentioned $\mathrm{C}$-arm inaccuracy, the results are very useful as it can be seen from the checker board representations of Fig. 4. Looking at the small veins in the registration result in of Fig. 4(b) we recognize an exact match.

\subsection{Robustness to Bad Initial Guesses}

We generated randomly 15 initial guesses and started from each of them 9 different registration problems: three different views on each of the three phantoms. The results were sorted according to their initial displacement that ranged from 
Table 1. Error table of the first experiment. Error values are the medians of the error distribution of all start positions given in $\mathrm{mm}$.

\begin{tabular}{|c|c|c|c|c|c|}
\hline Problem & Voting & BN - GC & BN - MI & BN - SSD & BN - PI \\
\hline \hline $3(\mathrm{a})$ & 3.5 & 3.5 & 26.6 & 4.2 & 25.0 \\
\cline { 1 - 1 } $3(\mathrm{~b})$ & 3.6 & 13.3 & 32.5 & 4.6 & 23.1 \\
\cline { 1 - 1 } $3(\mathrm{c})$ & 3.6 & 4.4 & 26.5 & 5.6 & 22.2 \\
\hline
\end{tabular}

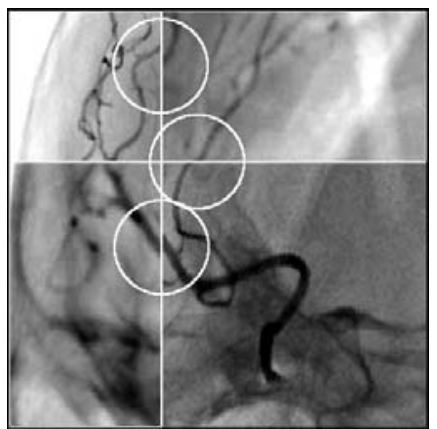

(a)

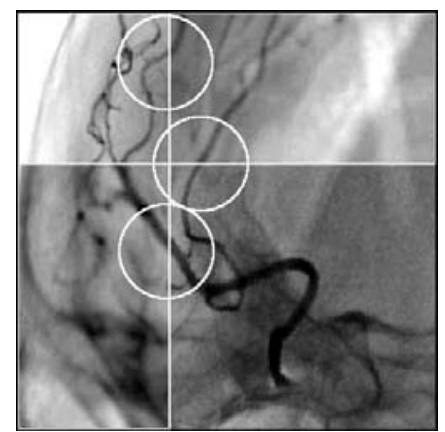

(b)

Fig. 4. Checker board representation of the system calibration (error $0 \mathrm{~mm})$ (a) and a registration result (b) which has an error value of more than $3 \mathrm{~mm}$

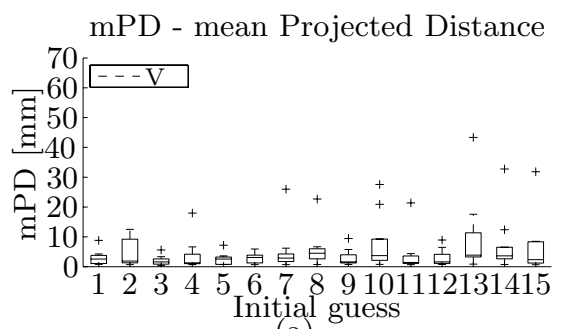

(a)

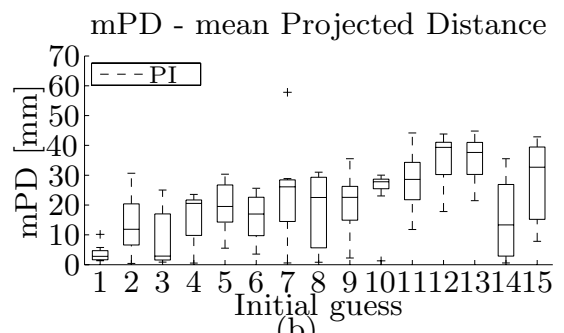

(b)

Fig. 5. Error distributions sorted by initial guesses for the vector optimization approach in (a) and a best neighbor approach with pattern intensity in (b) Boxes show 0.25-, $0.50-, 0.75$-quantiles, outliers are marked as points.

$10.2 \mathrm{~mm}$ up to $43.3 \mathrm{~mm}$. To our knowledge, this means an unpublished wide attraction range for medical image registration. Figure 5(a) shows the resulting error distribution beginning with the closest start position ' 1 ' and ending with the farthest one '15', where each distribution represents the results of all nine registration problems. Where the voting approach shows only very few outliers without a general drift to higher values, the best neighbor approach with PI shows a high sensitivity to local optima in Fig. (b).

\section{Discussion}

In this paper, we formalized the problem of medical image registration by means of vector optimization (1) and introduced a new algorithm that solves the problem 
accordingly. It makes use of multiple similarity measures and is therefore able to handle the problem of failing measures. Unlike other state of the art combination approaches, it avoids the summation of function values and by that the problem of single dominating similarity measures. Instead, in each iteration each similarity measure votes for an iteration direction according to its gradient information. This is a direct consequence of the working hypotheses that most of the similarity measures share a local optimum and therefore prefer the same iteration direction. The influence of each measure is controlled by dynamically adapted weights (2). Those use the iteration context by taking past iterations into account to detect failing measures automatically (4). By that strategy it is possible to get good registration results even with very bad initial guesses. An evaluation of the starting position showed almost constant result quality while increasing the initial error up to $43 \mathrm{~mm}$. To our knowledge, such high attraction ranges have not yet been published in medical image registration. Furthermore, the algorithm needs no datadependent modifications and solves the registration problem in a generic manner. This implies a high practical applicability for clinical applications. Future research topics will address the optimal choice of participating similarity measures to assure a democratic setup and the influence of history length for adapting the weights. A clinical evaluation will follow.

\section{References}

1. Livyatan, H., Yaniv, Z., Joskowicz, L.: Gradient-based 2-D/3-D rigid registration of flouroscopic X-ray to CT. IEEE Trans. on Med. Imaging 22(11), 1395-1405 (2003)

2. Viola, P.A.: Alignment by maximization of mutual information. International Journal of Computer Vision 24(2), 137-154 (2001)

3. Penney, G.P., Weese, J., Little, J.A., Desmedt, P., Hill, D.L.G., Hawkes, D.J.: A comparison of similarity measures for use in 2-D-3-D medical image registration. IEEE Trans. on Med. Imaging 17, 586-594 (1998)

4. Pluim, J., Maintz, J., Viergever, M.: Image registration by maximization of combined mutual information and gradient information. IEEE Trans. on Med. Imaging 19(8), 809-814 (2000)

5. Maintz, J., Viergever, M.: A survey of medical image registration. Medical Image Analysis 2(1), 1-36 (1998)

6. Garcia-Palomares, U.M., Gonzalez-Castaño, F.J., Burguillo-Rial, J.C.: A combined global \& local search (CGLS) approach to global optimization. Journal of Global Optimization 34(3), 409-426 (2006)

7. Zheng, G., Zhang, X.: A unifying MAP-MRF framework for deriving new point similarity measures for intensity-based 2D-3D registration. In: The 18th International Conference on Pattern Recognition, vol. 2, pp. 1181-1185 (2006)

8. Feldmann, T., Bouattour, S., Paulus, D., Deinzer, F.: Kombination verschiedener Ähnlichkeitsmaße für die 2D/3D-Registrierung von Röntgenbildern mittels Demokratischer Integration. In: Bildverarbeitung für die Medizin. Informatik Aktuell, pp. 226-230. Springer, Heidelberg (2006)

9. Triesch, J., von der Malsburg, C.: Democratic integration: Self-organized integration of adaptive cues. Neural Computation 13(9), 2049-2074 (2001)

10. Jahn, J.: Vector optimization - theory, applications, and extensions. Springer, Heidelberg (2004) 